\title{
Particulate Epidural Steroid may not be Detectable on MAgnetic Resonance IMAging
}

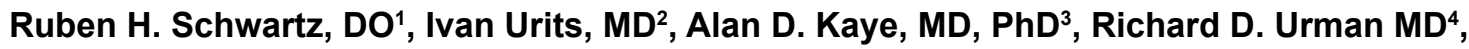 \\ Omar Viswanath, MD ${ }^{5-7}$, and Musa Aner, MD²
}

Lumbar epidural steroid injections (LESI) have become a staple in the interventional pain physician's armamentarium. For patients with radicular symptomatology, LESIs provide substantial pain relief with a favorable safety profile. Adverse effects, although rare, can occur as with any procedure. One of the most dreaded complications is the development of an epidural hematoma, which should be readily seen on Magnetic Resonance Imaging (MRI). It has been previously thought that particulate steroid injections should also be seen on MRI potentially obscuring a physician's clinical judgment. We present two cases where patients underwent an uncomplicated LESI and subsequently went for a lumbar MRI that displayed no injectate in the epidural space.

Key words: Epidural steroid injection, magnetic resonance imaging, particulate injection, hematoma
Lumbar epidural steroid injections (LESI) are safe and effective for the treatment of lumbar radicular pain (1). Complication rates from LESI are extremely low (2). In one study, 16,638 patients who underwent LESI were analyzed and found to have zero major adverse effects (3). Mass occupying lesions in the

From : ${ }^{1}$ Mount Sinai Medical Center, Department of Anesthesiology, Miami Beach, FL; ${ }^{2}$ Beth Israel Deaconess Medical Center, Department of Anesthesia, Critical Care, and Pain Medicine, Harvard Medical School, Boston, MA; ${ }^{3}$ Louisiana State University Health Sciences Center, Department of Anesthesiology, New Orleans, LA; ${ }^{4}$ Department of Anesthesiology, Perioperative and Pain Medicine, Brigham and Women's Hospital, Boston, MA; ${ }^{5}$ Valley Anesthesiology and Pain Consultants, Phoenix, AZ; ${ }^{6}$ University of Arizona College of Medicine - Phoenix, Department of Anesthesiology, Phoenix, AZ; ${ }^{7}$ Creighton University School of Medicine, Department of Anesthesiology, Omaha, NE

Author for correspondence: Ruben H. Schwartz, DO

Address: Mount Sinai Medical Center, Department of Anesthesiology, 4300 Alton Rd., Miami Beach, FL 33140

E-mail: rubenschwartz@yahoo.com

Disclaimer: There was no external funding in the preparation of this manuscript. Conflict of interest: Each author certifies that he or she, or a member of his or her immediate family, has no commercial association (i.e., consultancies, stock ownership, equity interest, patent/licensing arrangements, etc.) that might pose a conflict of interest in connection with the submitted manuscript. epidural space may result in neurologic deficit (4). It is thought that particulate steroid, deposited in the epidural space, may be visualized on magnetic resonance imaging (MRI) and subsequently make the diagnosis of underlying pathology challenging $(5,6)$.

Figure 1A demonstrates the procedural fluoroscopic imaging of an 80-year-old patient, with no risk factors for increased bleeding, who underwent an uncomplicated right $L 4 / 5$ interlaminar LESI for low back pain and lumbar radiculopathy. The injectate used included $80 \mathrm{mg}$ of methylprednisolone with local anesthetic for a total volume of $3 \mathrm{~mL}$. Figure $2 \mathrm{~A}$ demonstrates the procedural fluoroscopic imaging of a 79-year-old patient who underwent an uncomplicated caudal epidural injection for low back pain and lumbar radiculopathy, using a Coude needle and Brevi-Kath epidural catheter (Epimed, Dallas, TX). The injectate used included $2 \mathrm{~mL}$ of Depo-Medrol $40 \mathrm{mg} / \mathrm{mL}$ mixed with $2 \mathrm{~mL}$ of local anesthetic and sterile normal saline solution, which was deposited at the $L 4 / 5$ interspace level. In both cases, the patients underwent a previously scheduled MRI later that day after the LESI was performed. This was previously arranged due to an unrelated neurological deficit. Figures $1 \mathrm{~B}$ and $2 \mathrm{~B}$ demonstrate the respective MRIs acquired that 


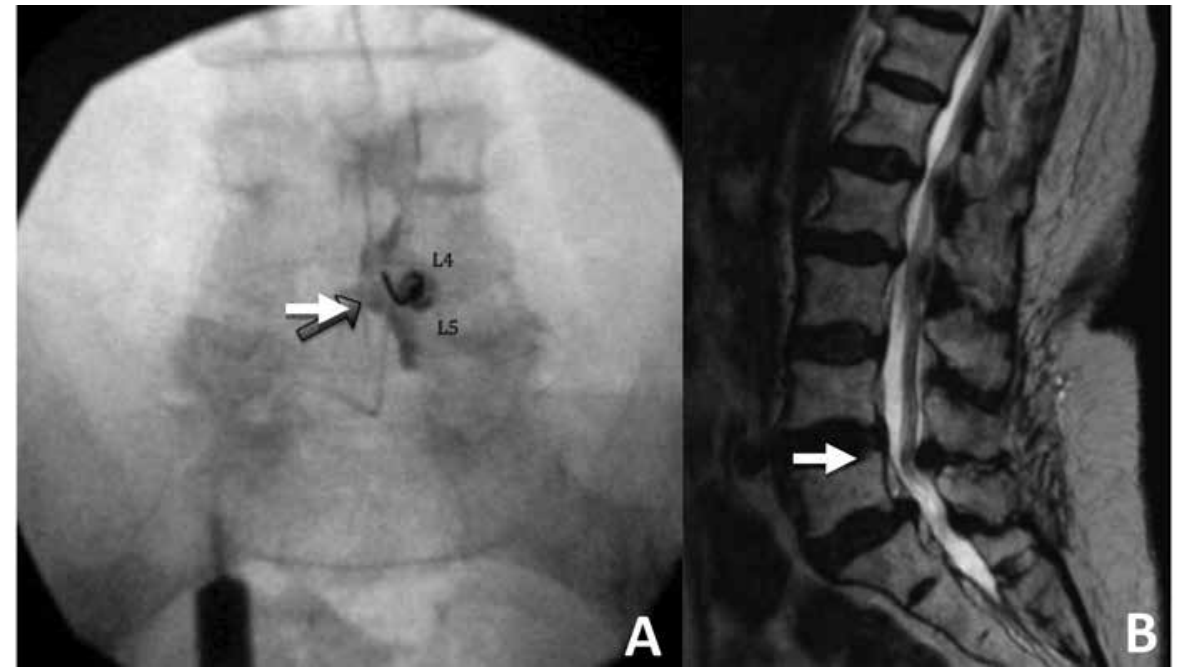

Fig. 1. (A) Intraoperative fluoroscopic imaging depicting epidural contrast medium spread (arrow) at the L4/5 level. (B) T2-weighted MRI imaging of the lumbar spine in the sagittal plane after interlaminar epidural injection demonstrating no evidence of an epidural collection.

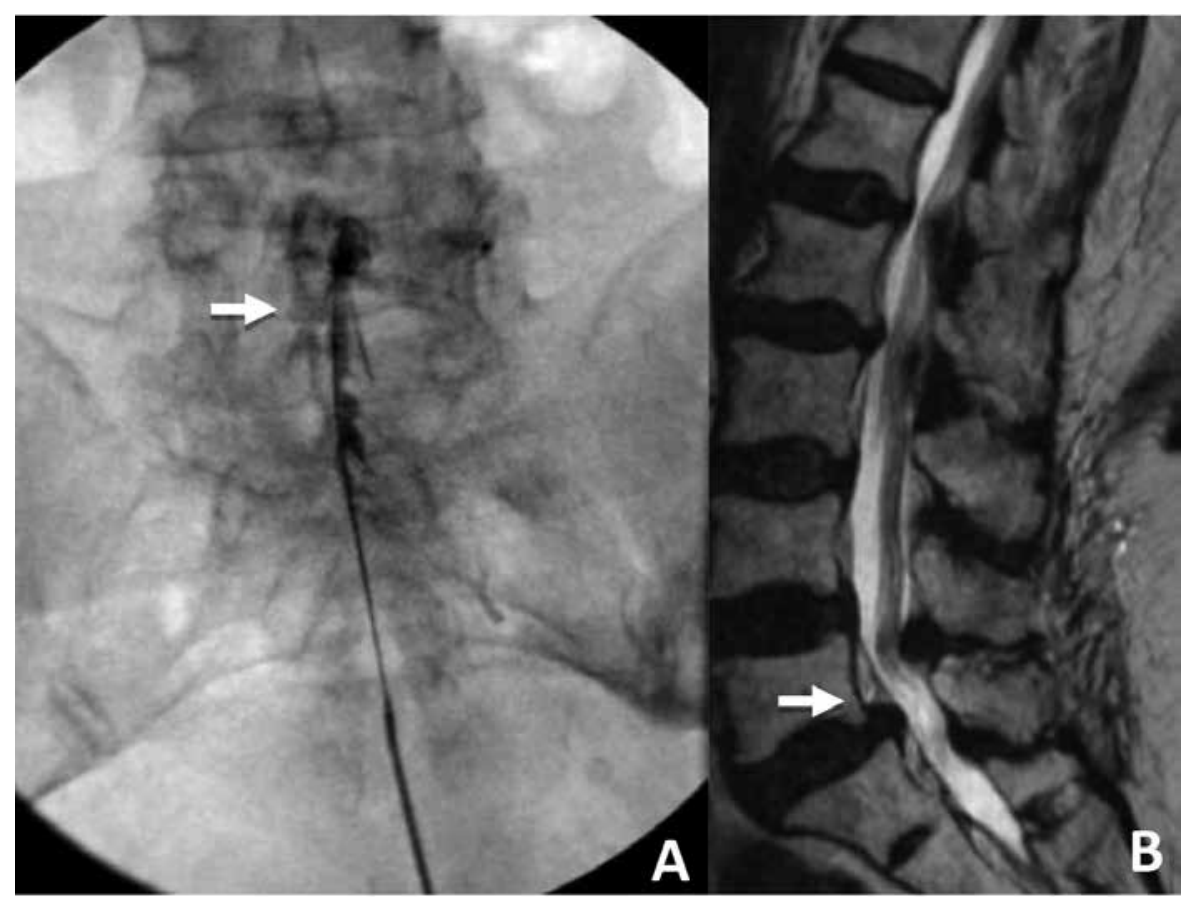

Fig. 2. (A) Intraoperative imaging depicting epidural contrast medium spread in the lumbar spine after caudal injection. (B) T2-weighted MRI imaging of the lumbar spine in the sagittal plane after caudal injection demonstrating no evidence of an epidural collection. demonstrated no evidence of epidural collection.

Our findings suggest that particulate steroid in the epidural space may not be detected on MRI. Further studies are needed to determine the sensitivity and specificity of MRI for the detection of particulate steroids in the epidural space. 
Particulate Epidural Steroid may not be Detectable on MRI

\section{REFERENCES}

1. Urits I, Burshtein A, Sharma M, et al. Low back pain, a comprehensive review: Pathophysiology, diagnosis, and treatment. Curr Pain Headache Rep 2019; 23:23.

2. Kaye AD, Manchikanti L, Abdi S, et al. Efficacy of epidural injections in managing chronic spinal pain: A best evidence synthesis. Pain Physician 2015; 18:E939-E1004.

3. El-Yahchouchi CA, Plastaras CT, Maus TP, et al. Adverse event rates associated with transforaminal and interlaminar epidural steroid injections: A multi-institutional study. Pain Med 2016; 17:239-249.

4. Sharma AK, Vorobeychik $Y$, Wasserman R, et al. The effective-

ness and risks of fluoroscopically guided lumbar interlaminar epidural steroid injections: A systematic review with comprehensive analysis of the published data. Pain Med 2017; 18:239-251.

5. Urits I, Israel B, Petro J, Asgerally A, Simopoulos T, Musa A. Conservative management of a cervical epidural hematoma after steroid injection article type: Case report. IPM Reports 2018; 2:213-219.

6. Kim MS, Jeong TY, Cheong YS, et al. Effect of epidural corticosteroid injection on magnetic resonance imaging findings. Korean J Pain 2017; 30:281-286.

IPM Reports Vol. 4, No. 1, 2020 
